\title{
EL ENTRENAMIENTO DE LA FUERZA Y EL MANTENIMIENTO DE LA SALUD DE MUJERES EN EDAD MENOPÁUSICA
}

DOI:105902/0102830812980

Data de submissão: 21/02/2014 Data de Aceite: 24/06/2014

\author{
Marcia Cristina Filippetto \\ Universidad de Lleida \\ marcia.filippetto@yahoo.com.br \\ Xavier Peirau Terés \\ Universidad de Lleida \\ xpeirau@inefc.es
}

RESUMEN: Esta investigación cuasi experimental fue realizada con una muestra $\mathrm{N}=43$ mujeres de edad intermedia de la ciudad de Lleida-España. Se ha basado en un marco teórico acerca de la importancia del ejercicio físico para el mantenimiento de la salud, planteando la realización de dos programas de ejercicio físico: acondicionamiento físico acuático y estimulación neuromuscular mecánica. El objetivo general fue conocer y comparar la influencia de los programas sobre la fuerza, como componente de la condición física, importante para las actividades de la vida diaria. Los programas tuvieran una duración de 12 semanas, con una frecuencia de dos entrenamientos a la semana. Se dispuso de un grupo control constituido por no practicantes de ejercicio físico y se ha aplicado el cuestionario IPAQ al inicio de la investigación para conocer la cantidad de actividad física realizada por la muestra. La fuerza fue evaluada mediante un test de pico máximo de fuerza isométrica realizado en tres momentos: basal, pre test y pos test. El análisis estadístico utilizó el método Anova para medidas repetidas y los resultados considerados significativos para $p<0,05$. Las practicantes de acondicionamiento físico acuático, estimulación neuromuscular mecánica y no practicantes de ejercicio físico $(n=17, n=13, n=13)$ no presentaron cambios significativos para la fuerza $(p=0,92, p=0,71, p=0,58)$.

Palabras clave: ejercicio físico, mujeres, fuerza muscular. 


\section{Introducción}

Las mujeres sufren una serie de transformaciones a nivel fisiológico en el periodo de la menopausia, esta se define como el cese permanente de la menstruación durante más de doce meses consecutivos en una mujer de más de 40 años de edad. Antes de esta edad se conoce con el nombre de menopausia precoz (ALVAREZ, MARTÍN \& BORDONES, 2008). Ocurre de media, alrededor de los 50 años de edad, aunque varía según factores genéticos y ambientales que pueden producir menopausias más tardías (KIRCHENGAST, 1993a, 1993b).

A partir de los 50 años las mujeres disminuyen la fuerza de prensión manual (SCHLUSSEL, DOS ANJOS, DE VASCONCELLOS \& KAC, 2008; VIANNA, OLIVEIRA \& ARAUJO, 2007) y la fuerza de las extremidades inferiores, más incluso que la fuerza de los brazos (LANDERS, HUNTER, WETZSTEIN, BAMMAN \& WEINSIER, 2001). Por este motivo dicha capacidad física debe ser trabajada de manera primordial en esta edad (CARBONELL, APARICIO \& DELGADO, 2009). Se aprecia una disminución del volumen y de la fuerza muscular llegando a tener dificultades para realizar tareas cotidianas que dependen de ella. Tal situación asociada a la disminución de la masa muscular resulta en una progresiva degeneración de la capacidad neuronal del individuo generando pérdida de la coordinación motora y de la propriocepción (MATSUDO, 2002).

El ejercicio físico (EF) es un agente importante en el mantenimiento de la salud, es una medida preventiva que interfiere sobre un número considerable de factores. Para su prescripción se deben considerar la intensidad, duración, frecuencia y progresión con el propósito de alcanzar un buen nivel de condición física y mejorar la salud mediante la reducción de los factores de riesgo de padecer de alguna enfermedad crónica (BECERRO, 1994; AMERICAN COLLEGE OF SPORTS MEDICINE, 7th ed.; 2006).

Dada la importancia del EF para el mantenimiento de la salud de la mujer en el periodo de la menopausia, abordamos dos diferentes programas de entrenamiento: acondicionamiento físico acuático (FA) y Estimulación Neuromuscular Mecánica (ENM). Por un lado están las prácticas acuáticas que tienen un importante índice de satisfacción comparadas con diferentes programas de entrenamiento físico terrestres y por otro lado se encuentra la ENM, una propuesta reciente con necesidad de mayor orientación profesional e investigación. 
El planteamiento de este estudio es conocer y comparar los efectos de ambos tipos de EF sobre la fuerza muscular, indispensable para el mantenimiento de las actividades de vida diaria (AVDs) de las mujeres en edad menopáusica. Dado que la fuerza condiciona una mejor composición corporal, con mayor porcentaje de masa libre de grasa y una incrementada densidad mineral ósea (AMERICAN COLLEGE OF SPORTS MEDICINE, 7th ed.; 2006) se reafirma su importancia en un programa de EF orientado hacia la salud de estas mujeres.

Actualmente se asocian los componentes de la condición física relacionada con la salud con un bajo riesgo de desarrollar prematuramente enfermedades derivadas del sedentarismo. Existe una estrecha relación entre los hábitos de vida, los niveles de actividad física y la condición física que determinan el estado de salud y calidad de vida de un individuo o población, como nos muestra la siguiente figura:

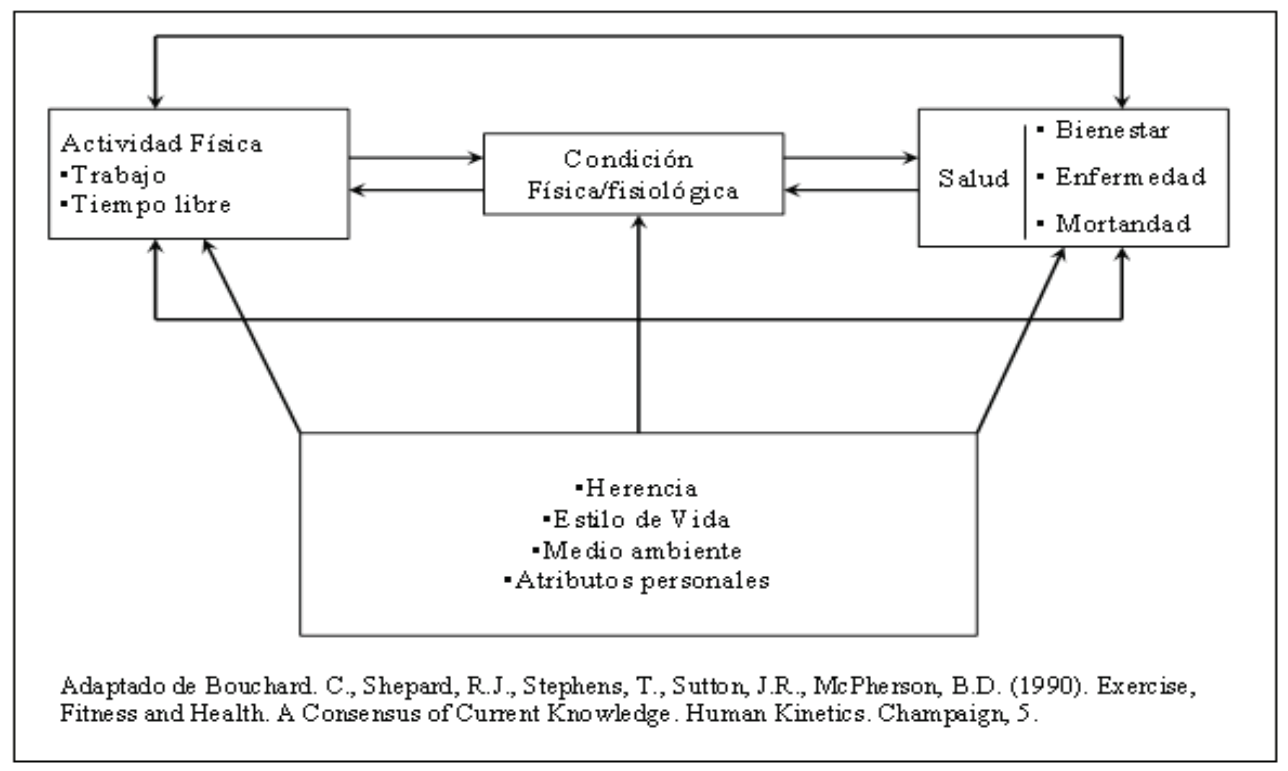

Figura 1: Interacciones entre actividad física, condición física y salud (BOUCHARD, SHEPHARD, STEPHENS, SUTTON Y MCPHERSON apud DELGADO \& TERCEDOR, 2002, p.29).

Bajo una perspectiva de salud pública, se indica el incremento de la actividad física de forma genérica, la manutención de un estilo de vida activo y la práctica de EF para el mantenimiento de la salud. Según Blair, Cheng \& Holder (2001), es preferible animar a las personas a ser físicamente activos en mayor medida que a tener una buena condición física, ya que es previsible que los sujetos sedentarios alcancen lo segundo si cumplen lo primero. 
Dentro de un universo de propuestas en lo que se refiere a práctica de EF, destacamos el entrenamiento de la fuerza como base para estas propuestas. El movimiento necesita una tensión muscular que se manifiesta por la aplicación de la fuerza. Tanto en el ámbito de la fisiología, de la biomecánica, o del entrenamiento deportivo el entrenamiento de la fuerza conlleva una mejora en la capacidad funcional de los practicantes y su evaluación permite adaptar los programas para mejor alcanzar los objetivos (BOSCO, 2000; GONZÁLEZBADILLO \& RIBAS, 2002).

La eficiencia de un programa de EF, según los objetivos propuestos, se refleja principalmente mediante la valoración de las capacidades físicas. La fuerza es una capacidad física que se relaciona significativamente con la realización de tareas diarias, ayuda a mantener y mejorar la movilidad articular y previene el riesgo de caídas y fracturas, sobretodo en la población femenina (DRINKWATER, 2006; MATSUDO, 2008).

Tomando como directriz la prescripción de EF para el mantenimiento de la salud, nos preguntamos ¿Cuáles son los efectos de los programas de FA y ENM en la fuerza de las mujeres en edad menopáusica?

\section{Metodología}

Los programas de EF se desarrollaran en 25 sesiones, con una frecuencia de dos veces a la semana por un período de tres meses.

La población fue constituida por personas adultas del género femenino con edad intermedia de la ciudad de Lleida practicantes de FA, ENM y no practicantes de EF (grupo control).

Las componentes de la muestra firmaron un consentimiento informado de libre participación, conforme no presentaban ninguna enfermedad diagnosticada que incapacitara la realización de EF y contestaran el cuestionario IPAQ (International Physical Activity Questionnaire, forma corta, auto-administrado, versión 2002) para realizar el registro de la cantidad de actividad física inicial de las mismas.

\section{Caracterización de la muestra}

La composición de la muestra siguió los criterios de inclusión y exclusión de acuerdo con la figura: 


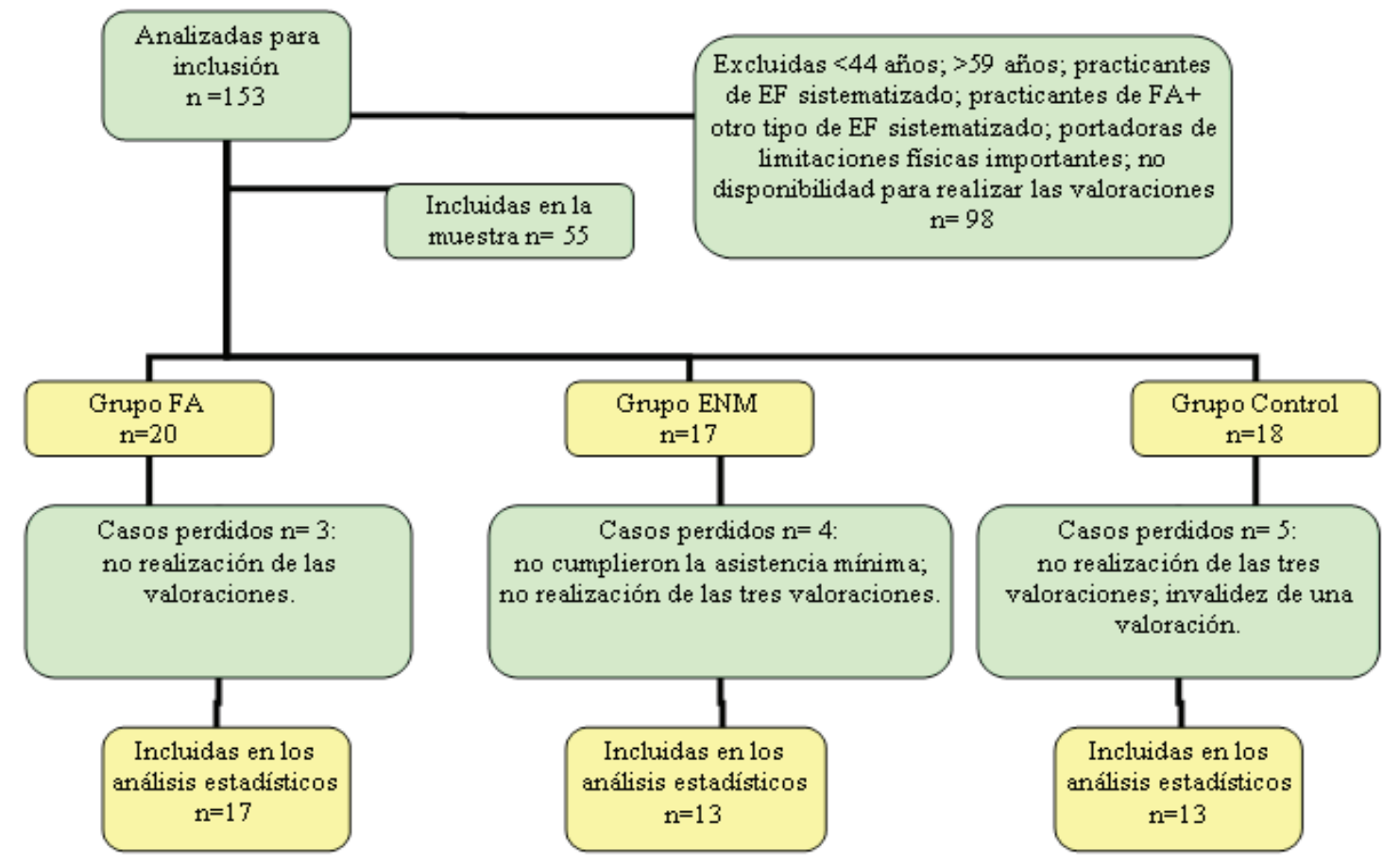

Figura 2: Descripción de la muestra.

A parte de la valoración de la fuerza, también se han considerado para el análisis estadístico las variables modificadoras: edad, peso, talla, índice de masa corporal (IMC) y asistencia a las sesiones.

\section{Caracterización de los programas de EF}

Los grupos obedecieran la misma frecuencia y duración evolucionando la intensidad de acuerdo con las características de cada programa.

\section{-Acondicionamiento físico acuático (FA)}

Los contenidos desarrollados fueron actividades de predominancia aeróbica; juegos lúdico-sociales; ejercicios de tonificación; actividades de relajación; ejercicios de flexibilidad; actividades de consciencia y autocontrol de la intensidad del ejercicio; running en aguas profundas; trabajo interválico y circuitos. Hemos adoptado una mezcla de estos contenidos, según el momento de cada sesión (calentamiento, parte principal, vuelta a calma) y el objetivo específico para cada una de ellas. Las progresiones a lo largo del programa se hicieron a nivel de intensidad controlada por la Escala de Borg modificada (con base en NOBLE, BORG, JACOBS, CECI \& KAISER [1983] apud ACSM, [2005]). 
La primera sesión fue de "familiarización" con el ambiente acuático, con los materiales utilizados, con el instrumento de percepción del esfuerzo (Escala de Borg modificada) y con el grupo. Las primeras 12 sesiones el entrenamiento estuvo dentro de un rango de intensidad ligera-moderada y las ultimas 12 sesiones dentro de un rango de intensidad moderada, siguiendo las recomendaciones del American College of Sports Medicine (ACSM, 2005) para mujeres adultas con edad intermedia.

\section{-Estimulación neuromuscular mecánica (ENM)}

El entrenamiento estuvo condicionado a las posibilidades de utilización de la plataforma vibratoria utilizada (Fitvibe Medical) en lo que se refiere a las variaciones de frecuencia, amplitud, nivel y tiempo con la progresión de las mismas de acuerdo con los ejercicios y semana de entrenamiento. Se han modificado las variables frecuencia, entre 20 y $40 \mathrm{~Hz}$; amplitud, entre dos y cuatro milímetros (mm); tiempo, entre 30 y 55 s para cada ejercicio y nivel de entrenamiento (Inicial, Intermedio y Avanzado).

El nivel Inicial consistió en una frecuencia entre 20 y $35 \mathrm{~Hz}$, tiempo de 30 a 45s, amplitud de dos mm con cuatro ejercicios. El nivel Intermedio, una frecuencia entre 30 y $38 \mathrm{~Hz}$, tiempo entre 30 y 55s, amplitud de dos $\mathrm{mm}$ con cinco ejercicios en las dos primeras semanas, tres en estática y dos en dinámica; y cuatro ejercicios en las cinco semanas siguientes, dos en estática y dos en dinámica. El nivel Avanzado, una frecuencia entre 30 y $40 \mathrm{~Hz}$, tiempo entre 30 y 40 s y amplitud de cuatro $\mathrm{mm}$.

Entre un ejercicio y otro se utilizó un tiempo de recuperación constante de 60 segundos (MARIN, [2008]; DE HOYO, GRANADOS, CARRASCO \& SAÑUDO, [2009]). Se optó por realizar un programa con duración de un mes a un nivel Inicial, con la frecuencia, tiempo y amplitud recomendados por el programa estándar de la plataforma vibratoria utilizada. Los valores de la frecuencia y amplitud coincide con el estudio de Roelants, Delecluse, Goris \& Verschueren (2004), que presentó resultados favorables en relación al aumento de la fuerza en personas de edad intermedia.

Los dos meses siguientes las sesiones se han realizado a un nivel Intermedio, con una frecuencia entre 30 y $35 \mathrm{~Hz}$, utilizada en los estudios de Torvinen et al. (2002) y Roelants, Delecluse, Goris \& Verschueren (2004). También está de acuerdo con la recomendación 
de Fajardo \& Ferliú (2004), entre 23 y 44 Hz y de Marin (2008), entre 23 y 47 Hz. El tiempo utilizado fue más bajo que los estudios de Fagnani, Giombini, Di Cesare, Pigozzi \& Di Salvo (2006) y Torvinen et al. (2002) pues estos se aplicaron a deportistas o jóvenes.

Únicamente en la última sesión se pasó a un nivel Avanzado porque la literatura no tiene claramente definidos parámetros ideales para cada tipo de población (MARÍN, 2008).

Tras la aplicación de estos programas de EF, nos interesa evaluar su incidencia sobre la fuerza de los sujetos, para tal González-Badillo \& Ribas (2002), recomienda la medición del pico de fuerza conseguido y el tiempo necesario para llegar a alcanzarlo (relación fuerza $x$ tiempo). El pico máximo de fuerza (PMF), se mide cuando no hay movimiento, que se caracteriza por la fuerza isométrica máxima, también denominada fuerza estática. El mismo autor destaca que la medición consiste en la aplicación de la fuerza máxima voluntaria a una resistencia insuperable.

La medición se consigue con fiabilidad y precisión con un "transductor" de fuerza (célula de carga) y un soporte informático como es el sistema MuscleLab (Bosco System ® 4000 - Ergotest Technology versión 7.18, Noruega). Se conecta un sensor de movimiento a la carga a desplazar y el equipo registra el desplazamiento en función del tiempo de forma numérica y gráfica.

Hemos medido el PMF de los músculos extensores de la rodilla alcanzado con una resistencia insuperable y ausencia de movimiento (fuerza isométrica) por medio del Musclelab. Para tal, hemos utilizado una prensa horizontal con la célula de medición de fuerza acoplada y conectada a un ordenador portátil con el software. Para que la fuerza aplicada por la participante no superara la resistencia, se adaptó una cadena acoplada a la célula de carga que impidió el movimiento en el momento de empuje. La duración del esfuerzo máximo fue de cinco segundos con un ángulo de las rodillas variando entre 120 y 125 grados.

Las mediciones se realizaron previamente a los programas de EF, con una valoración basal, un pre test y posteriormente a los programas de EF (pre y pos test). En cada una de ellas se hicieran tres repeticiones y de estas se eligió el valor más elevado para el análisis estadístico realizado con el Statistical Package for Social Science (SPSS) versión 15.0. 


\section{Resultados y Discusión}

En este apartado analizamos los efectos de los programas de entrenamiento a partir de comparaciones entre las variables dependientes y las posibles variables modificadoras del efecto.

Las variables modificadoras valoradas en el pre test y en el pos test son el peso y el IMC, donde ambas no presentaron diferencias estadísticamente significativas. Para el IMC, hemos clasificado la muestra según los índices de normalidad presentados por la OMS entre Normal, Sobrepeso y Obesidad (LEERMAKERS, DUNN \& BLAIR, 2000) y no se han observado cambios en los mismos.

La asistencia fue mencionada en el apartado descripción de la muestra, pues se consideró como un criterio de inclusión y exclusión. La edad y la talla solamente han sido valoradas una vez, dada la corta duración de los programas de entrenamiento. La variable nivel de actividad física valorada inicialmente para los grupos de FA y ENM y mensualmente para el grupo Control.

También se consideran las diferencias estadísticamente significativas para la edad $(p=$ $0,01)$ y la asistencia $(p<0,01)$, aunque, debido al tamaño limitado de la muestra, podría ser que no hubiera suficiente potencia estadística para detectarlas. Se ha aplicado el análisis multivariado y los resultados han mostrado que la interacción entre estas variables en el tiempo no fue estadísticamente significativa, por este motivo no se incluyeron en el modelo estadístico.

Se analizaran los resultados de dos maneras: comparando la variable entre los grupos (intergrupos) y comparando la variable dentro del mismo grupo (intragrupos).

El análisis intergrupos nos permite conocer las características iniciales de cada grupo, si existen diferencias entre ellos y si evolucionan de manera similar desde la valoración basal al pre test. De manera que los resultados encontrados en el pos test se deban al entrenamiento y no a una diferencia inicial entre los grupos.

El análisis intragrupo se compone de dos momentos, el primero consiste en la comparación entre la valoración basal y el pre test para cada grupo. Donde se verifica si estos valores iniciales de las variables no se deben a un aprendizaje en la ejecución de los tests. Para identificar o descartar tal aprendizaje se aplicó la prueba paramétrica 
t-Student para muestras relacionadas con los valores reales de las variables, y la prueba no paramétrica Wilcoxon para muestras relacionadas, con los rangos de las variables. El segundo momento del análisis intragrupo fue la comparación a lo largo del tiempo, entre el pre test y el pos test, para comprobar los efectos de los programas de entrenamiento y del no entrenamiento (grupo control).

Con estos análisis, se comprobó que la fuerza de las mujeres de edad intermedia no ha presentado variación significativa tras la aplicación de ambos programas de EF y no practicantes de EF, tal como representa la tabla a continuación:

Tabla 1: Variación de la fuerza - pre y pos tests de cada grupo.

\begin{tabular}{ccccccccccccc}
\hline & \multicolumn{3}{c}{ Grupo FA } & & Grupo ENM & & Grupo Control & p \\
intergrupos
\end{tabular}

Nota: Valor $p$ de comparación intergrupos obtenido por Anova de un factor. Valor $p$ de comparación intragrupos obtenido por el modelo lineal general para medidas repetidas.

Los valores de $p>0,05$ apuntan hacia una evolución similar de la fuerza en los tres grupos, aunque se hayan sometido a entrenamientos distintos y al no entrenamiento (control).

Los niveles de fuerza superiores detectados para el grupo Control podrían estar relacionados con el nivel inicial de actividad física registrado por el IPAQ, donde hemos constatado que $77 \%$ de las participantes de este grupo estaban a un nivel moderado de actividad física y $23 \%$ a un nivel bajo, y así lo mantuvieron a lo largo de los tres meses. En cambio en los grupos de FA y ENM, 47\% de las participantes estaban a un nivel moderado y $53 \%$ a un nivel bajo al principio de los programas de entrenamiento. Un factor que podría influir en que el grupo Control presente unos niveles de fuerza superiores respecto a los otros grupos es la menor edad de sus componentes, de acuerdo a las diferencias estadísticamente 
significativas encontradas en la comparación intergrupos. Aunque al valorarla en el modelo estadístico no se haya observado diferencias significativas en el efecto, podría influir sin la suficiente potencia para que se detecte como significativa.

De la misma forma el grupo Control no ha presentado decremento, que sería más evidente por la no práctica de EF sistematizado. Se podría atribuir al nivel de actividad física superior que presentaban al principio, cuando fueron comparadas con los otros grupos, sus AVD se mantuvieron estables y con ello sus niveles de fuerza.

Estos resultados nos conducen a una evaluación de la eficacia de los programas de EF tales como han sido desarrollados para esta población. Así, presentamos las siguientes consideraciones acerca de cada uno de ellos:

\section{Programa de FA}

Dado que el medio acuático es un lugar donde constantemente se tiene que vencer la resistencia al movimiento, consecuentemente es necesario aplicar más fuerza (COLADO, 2008), lo que conduce por si solo al desarrollo de la fuerza resistencia. El desarrollo de la fuerza máxima sería un paso que podría ser dado si el programa de acondicionamiento estuviera direccionado al rendimiento deportivo (CASPERSEN, POWELL \& CHRISTENSON, 1985; PATE, 1983). En este caso, un programa de acondicionamiento direccionado al rendimiento posiblemente no motivaría la asistencia de estas mujeres, considerados los estudios de Navarro et al. (2008), que dice que las mujeres buscan en el EF un bienestar integral como fin y no como medio y de Colado (2008), que destaca la mejora en la relación social y la diversión de sus ejercitantes. El programa desarrollado indica tales características y por tal motivo tiene buena aceptación.

La relación entre la fuerza y la condición física general (BOSCO, 2000) planteada en este estudio está orientada hacia una perspectiva de mantenimiento y promoción de la salud. Las características de este programa de EF apuntan a que se desarrolle la resistencia a la fuerza, donde la tensión muscular es relativamente prolongada sin que disminuya la efectividad de la misma. Los estudios de Emslander et al. (1998) y Thein \& Brody (1998) apud Colado \& Moreno (2001), confirman que hay mejora de la resistencia a la fuerza y de su manifestación activa con el entrenamiento acuático. 
Destacamos la importancia de la fuerza, así como de la fuerza resistencia para esta población, como un factor favorecedor de la condición física general, mejorando la funcionalidad en las AVD (JIMÉNEZ, 2003; CASPERSEN, POWELL \& CHRISTENSON, 1985; BLAIR, CHENG \& HOLDER, 2001), contribuyendo en la prevención de caídas (GUSI, RAIMUNDO \& LEAL, 2006; DRINKWATER, 2006; MATSUDO, 2008) y en el mantenimiento y prevención de la salud (BAÑUELOS, 1996; BOUCHARD, SHEPHARD, STEPHENS, SUTTON Y MCPHERSON apud DELGADO \& TERCEDOR, 2002).

\section{Programa de ENM}

Los efectos de este programa de EF sobre los niveles de condición física y salud son poco conocidos (GUSI, RAIMUNDO \& LEAL, 2006; DE HOYO, GRANADOS, CARRASCO \& SAÑUDO, 2009), pues presenta varios parámetros que pueden ser modificados como son la frecuencia, la amplitud, la dirección o duración de la vibración, tiempo de descanso y causar distintos efectos, de acuerdo con Moras, Tous, Muñoz, Padullés \& Valejjo, (2006) y Marin (2008). Su relación con la fuerza tiene muchas variantes presentadas en otros estudios, como el de Roelants, Delecluse, Goris \& Verschueren, (2004) con mujeres postmenopáusicas que presentó un aumento de la fuerza isométrica de los músculos extensores de la rodilla, tras un programa de 24 semanas con una frecuencia inicial de 35 $\mathrm{Hz}$ y una duración de 20 a 30 min, todos estos parámetros son superiores a los aplicados en esta investigación, posible motivo del incremento de la fuerza.

Otro factor que puede interferir en los efectos del programa de ENM es la opción "miembros inferiores", seleccionada para esta investigación. A diferencia de los estudios de Delecluse, Roelants \& Verschueren (2003) y Torvinen (2002), que utilizaron la opción "todo el cuerpo" resultando un incremento en la fuerza. En cambio, Fernandez-Rio, Terrados, Fernandez-Garcia \& Suman (2010) concluye que el ENM de todo el cuerpo no supone ninguna ventaja sobre los métodos tradicionales de entrenamientos de la fuerza en jugadoras de baloncesto. Por ello considera que la opción de miembros inferiores o todo el cuerpo no es un factor decisivo para la obtención de un resultado u otro.

Coincidimos con Marin (2008), cuando dice que la ENM no debe ser vista como substitutivo de un entrenamiento convencional, sino como un complemento a otras actividades 
o programas de EF, incluidas las AVD. La asistencia a los entrenamientos de ENM fue un factor favorable en los resultados, la aceptación de un tipo de EF desconocido para esta muestra, apunta la posibilidad de aplicar este programa como complemento a otros tipos de EF. Con la posibilidad de mantener o ajustar los parámetros utilizados, según objetivos deseados y teniendo como un factor favorable el tiempo reducido de aproximadamente 30 minutos, para la realización de cada sesión.

\section{Consideraciones finales}

El vacío de información específica respecto a qué programa de EF es el más indicado para las mujeres en esta edad nos llevó a trazar los objetivos de esta investigación, comparando dos programas distintos de EF y sus efectos sobre la fuerza y su relación directa con las AVDs. Los resultados encontrados para esta población no nos permiten hacer una inferencia a todas las mujeres de edad intermedia que realizan estos programas

de $E F$, debido a nuestra limitación en la composición de la muestra (grupo $F A=17$, grupo $\mathrm{ENM}=13$ y grupo Control=13).

Tales resultados pueden ser auxiliares en la elección de un tipo de EF, ya que depende de diversos factores, como son la preferencia por las características del tipo de EF, necesidades y limitaciones personales, objetivos con la práctica, disponibilidad de tiempo, comodidad en relación al material necesario para las prácticas, nivel inicial de actividad física, diferencias sociales, feedback aplicado, experiencias deportivas previas, presupuesto, entre otros. Todo el conjunto de factores conduce a la elección por el programa "más adecuado" de EF direccionado hacia la salud, para las mujeres en esta etapa de la vida.

El presente estudio está orientado hacia la manutención y promoción salud, así la valoración de la fuerza nos interesa para conocer la existencia de cambios no solamente desde un punto de vista de incremento de fuerza sino del mantenimiento de la misma a lo largo del tiempo. Si el nivel de fuerza se mantiene a lo largo del período de aplicación de los programas de EF, se puede considerar un factor positivo, dada la edad de las participantes y el proceso natural de pérdida de la condición física en que se encuentran. 
A partir de tales resultados, se presentan posibilidades para futuras investigaciones, modificando por ejemplo, la "dosis" utilizada e duración de los programas de EF. El entrenamiento de la fuerza dentro de estos programas tiene objetivos distintos a los de un entrenamiento orientado hacia el rendimiento deportivo. Bajo esta perspectiva, concordamos con Heredia Elvar, Costa \& Abril (2005), cuando dice que la valoración de la fuerza debe servir para determinar la zona de entrenamiento neuromuscular en la que un sujeto debe iniciar un programa, garantizar una transferencia directa entre los datos obtenidos y su aplicación a la prescripción. Todo ello debe realizarse evitando situaciones que supongan riesgo de lesiones; permitiendo la comprobación de la evolución y de los efectos del entrenamiento.

\section{Referencias}

AlVAREZ, R. G.; MARTíN, E. G.; BORDONES, M. A. Conocimiento y actitud sobre el climaterio en mujeres entre 40 y 50 años. Rev Obstet Ginecol.Venez, 68(1), 32-40, (2008).

AMERICAN COLLEGE OF SPORTS MEDICINE. Manual ACSM para la valoración y prescripción del ejercicio. 2. ed. Barcelona: Paidotribo, 2005.

AMERICAN COLLEGE OF SPORTS MEDICINE. ACSM's Guidelines for Exercise Testing and Prescription. 7th ed. Baltimore: Lippincott Williams \& Wilkins, 2006.

BAÑUELOS, F. S. La actividad física orientada hacia la salud. Madrid: Biblioteca Nueva, S. L., 1996.

BLAIR, S. N.; CHENG, Y.; HOLDER, J. S. Is physical activity or physical fitness more important in defining health benefits? Medicine and Science in Sports and Exercise, 33(6),S379-S99, 2001.

BOSCO, C. La fuerza muscular. Barcelona: INDE, 2000.

CARBONELL, A. B.; APARICIO, V. G.; DELGADO, M. F. Efectos del envejecimiento en las capacidades físicas: implicaciones en las recomendaciones de ejercicio físico en personas mayores. Rev. Int. Cienc. Deporte, 17(5), 1-18. doi: 10.5232/ricyde2009.017.0, 2009. 
CASPERSEN, C. J. ; POWELL, K. E. ; CHRISTENSON, G. M. Physical activity, exercise and physical fitness: definitions and distinsctions for health-related research. Public Health Nutr, 100, 126-131, 1985.

COLADO, S. J. C. Criterios metodológicos para el entrenamiento de la fuerza en el medio acuático. En JIMÉNEZ GUTIERREZ, A. (Ed.), Nuevas Dimensiones en el entrenamiento de la Fuerza: aplicación de nuevos métodos, recursos y tecnologías. (pp. 383-418). Barcelona: INDE, 2008.

DE HOYO, M.; GRANADOS, S. R.; CARRASCO, L.; SAÑUDO, B. Revisión del efecto agudo de las vibraciones mecánicas sobre diversas manifestaciones de la fuerza. Revista Archivos de Medicina del Deporte, 26(129), 14-21, 2009.

DELECLUSE, C.; ROELANTS, M.; VERSCHUEREN, S. Strength increase after wholebody vibration compared with resistance training. Med Sci Sports Exerc, 35(6), 1033-41, 2003.

DELGADO, M. F.; TERCEDOR, P. S. Estrategias de intervención en educación para la salud desde la educación física. Barcelona: INDE, 2002.

DRINKWATER, B. L. El Ejercicio y la Mujer Posmenopáusica. PubliCE Standard. Pid: $644,2006$.

FAJARDO, J. T.; FERLIÚ, G. M. Entrenamiento por medio de vibraciones mecánicas: revisión de la literatura. Revista Digital, Educación Física y Deportes, 10, 79, 2004. Recuperado de http//:www.efdeportes.com, (Consulta en 18/05/2008).

FERNANDEZ-RIO, J.; TERRADOS, N.; FERNANDEZ-GARCIA, B.; SUMAN, O. E. Effects of Vibration Training on Force Production in Female Basketball Players. J Strength Cond Res, 24(5), 1373-138, 2010. doi: 10.1519/JSC.0b013e3181d1d2b1

FILIPPETTO, M. C. ; OLASO, S. C. Incidencia de la fuerza en los músculos extensores de la rodilla en mujeres de 45 a 55 años practicantes de actividad físico deportiva de mantenimiento. Revista Digital, Educación Física y Deportes, 122. Recuperado de http:// www.efdeportes.com, (Consulta en: 12/05/11).

FAGNANI, F.; GIOMBINI, A.; DI CESARE, A.; PIGOZZI, F.; DI SALVO, V. The effects of a whole-body vibration program on muscle performance and flexibility in female athletes. Am J Phys Med Rehabil, 85, 956-962, 2006. 
GONZÁLEZ-BADILLO, J. J.; GOROSTIAGA, E. Fundamentos del entrenamiento de la fuerza. Barcelona. INDE, 1995.

GONZÁLEZ-BADILLO, J. J.; RIBAS, J.S. Bases de la programación del entrenamiento de fuerza. Barcelona. INDE, 2002.

GUSI, N.; RAIMUNDO, A.; LEAL, A. Low-frequency vibratory exercise reduces the risk of bone fracture more than walking: a randomized controlled trial. BMC Musculoskeletal Disorders, 7(92), 2006. doi:10.1186/1471-2474-7-92.

JIMÉNEZ, A. Fuerza y Salud, la aptitud músculo-esquelética, el entrenamiento de fuerza y la salud. Barcelona: Ergo, 2003.

KIRCHENGAST, S. Relations between anthropometric characteristics and degree of severity of the climateric syndrome in austrian women. Maturitas, 17, 167-80, 1993a.

KIRCHENGAST, S. Anthropometric-hormonal correlation patterns in fertile and postmenopausal women from Austria. Ann Hum Biol, 26, 47-65, 1993b.

LANDERS, K. A.; HUNTER, G. R.; WETZSTEIN, C. J.; BAMMAN, M. M.; WEINSIER, R. L. The interrelationship among muscle mass, strength, and the ability to perform physical tasks of daily living in younger and older women. Journal of Gerontology: Medical Sciences, 56(10), B443-448, 2001.

LEERMAKERS, E.; DUNN, A.; BLAIR, S. Exercise management of obesity. Med Clin N Am, (84), 1-19, 2000.

MARCOS BECERRO, J. F. Ejercicio, forma física y salud: fuerza, resistencia y flexibilidad. Madrid: Eurobook, 1994.

MARIN, P. J. Aplicaciones de la estimulación neuromuscular mecánica (vibraciones): recursos metodológicos y tecnológicos. En JIMÉNEZ GUTIERREZ, A. (Ed.), Nuevas Dimensiones en el entrenamiento de la Fuerza: aplicación de nuevos métodos, recursos y tecnologías (271-308). Barcelona: INDE, 2008.

MATSUDO, S. M. Envelhecimento, atividade Física e saúde. R. Min. Educ. Fís, 10(1), 195-209, 2002.

MATSUDO, S. M. Atividade Física: Passaporte para a Saúde. Motriz Rio Claro, 14(2), S12-13. (Supl. II Congresso Internacional de Biodinâmica da Unesp, p.S1-S141), 2008. 
MORAS, G.; TOUS, J.; MUÑOZ, C. J.; PADULLÉS, J. M.; VALEJJO, L. Electromyographic response during whole-body vibrations of different frequencies with progressive external loads. Revista Digital, Educación Física y Deportes, 10, 93, 2006. Recuperado de http//:www.efdeportes.com, (Consulta en 13/10/2008).

NAVARRO, J. E.; GONZÁLEZ RIVERA, M. D.; RODRÍGUEZ, M. M.; PINÉS, D. H.; CASTILLO, J. M. Hábitos de actividad física y demandas de servicios de actividad física de las mujeres adultas. Revista Digital, Educación Física y Deportes, 12, 118, 2008. Recuperado de http//:www.efdeportes.com, (Consulta en 10/10/2011).

NOBLE, B.J.; BORG, G.A.V.; JACOBS, I.; CECI, R; KAISER, P. A category-ratio perceived exertion scale: relationship to blood and muscle lactates and heart rate. Medicine and Science in Sports and Exercise, 115(6), 523-528, 1983.

PATE, R. R. A new definition of youth fitness. The Physician and Sports Medicine; 11, 77-83, 1983.

ROELANTS, M.; DELECLUSE, C.; GORIS, VERSCHUEREN, S. M. Effects of 24 weeks of whole body vibration training on body composition and muscle streght in untrained females. Int J Sports Med(25), 1-5, 2004.

SCHLUSSEL, M. M.; DOS ANJOS, L. A.; DE VASCONCELLOS, M. T.; KAC G. Reference values of handgrip dynamometry of healthy adults: a population-based study. Clin Nutr, 27(4), 601-7, 2008. doi:10.1016/j.clnu.2008.04.004

TORVINEN, S.; KANNUS, P.; SIEVANEN, H.; JARVINEN, T. A.; PASANEN, M.; KONTULAINEN, S.; JARVINEN, T. L.; JARVINEN, M.; OJA, P.; VUORI, I. Effect of fourmonth vertical whole body vibration on performance and balance. Med Sci Sports Exerc, 34, 1523-8, 2002.

VIANNA, L. C.; OLIVEIRA, R. B.; ARAÚJO, C. G. S. Age-Related Decline in Handgrip Strength Differs According to Gender. J. Strength Cond. Res, 21(4), 1310-1314, 2007. 


\section{O TREINAMENTO DE FORÇA E MANUTENÇÃO DA SAÚDE DAS MULHERES EM IDADE DE MENOPAUSA}

RESUMO: Esta investigação quase-experimental foi desenvolvida com uma amostra de $\mathrm{N}=43$ mulheres de idade intermediária da cidade de Lleida-Espanha. Baseou-se em um marco teórico sobre a importância do exercício físico para a manutenção da saúde, propondo a realização de dois programas de exercício físico: condicionamento físico aquático e estimulação neuromuscular mecânica. O objetivo geral foi conhecer e comparar a influência dos programas sobre a força, como componente da condição física, importante para as atividades de vida diária. Os programas tiveram uma duração de 12 semanas, com uma frequência de dois treinos por semana. Dispôs-se de um grupo controle de não praticantes de exercício físico e aplicou-se o questionário IPAQ no início da investigação para conhecer a quantidade de atividade física da amostra. A força foi avaliada através de um teste de pico máximo de força isométrica realizado em três momentos: basal, préteste e pós-teste. A análise estatística utilizou o método Anova para medidas repetidas e os resultados considerados significativos para $p<0,05$. As mulheres praticantes de condicionamento físico aquático, estimulação neuromuscular mecânica e não praticantes de exercício físico ( $n=17, n=13, n=13)$ não apresentaram mudanças significativas para a força $(p=0,92, p=0,71, p=0,58)$.

Palavras chave: exercício físico, mulheres, força muscular. 


\section{THE STRENGTH TRAINING AND MAINTAINING THE HEALTH OF WOMEN OF MENOPAUSAL AGE}

ABSTRACT: This quasi-experimental research has been conducted with a sample $\mathrm{N}=43$ of middle-aged adult women in the city of the Lleida-Spain. Based on a theoretical framework about the importance of exercise for maintaining the health of this population, we propose leading two training programs: water fitness and neuromuscular stimulation mechanic. The overall objective is to know and compare the influence of training programs on strength, components of fitness needed for everyday activities. Training programs have duration of 12 weeks, with a frequency of two workouts per week. Parallel have a control group of non-practitioners exercise. IPAQ questionnaire is applied early in the investigation to determine the amount of physical activity undertaken by the sample.The strength was evaluated by a test of peak isometric strength carried out in three assessments: baseline, pre-test and post-test. Statistical analysis used repeated measures Anova method and the results considered significant at $p<0,05$. Women aquatic fitness practitioners, neuromuscular stimulation mechanical practitioners and do not do exercise practitioners $(n=17, n=13, n=13)$ do not make significant changes for strength $(p=0,92, p=0,71, p=0,58)$.

Keywords: exercise, women, strength muscle. 